\title{
Bihamiltonian Geometry and Separation of Variables for Toda Lattices
}

\author{
Gregorio FALQUI $\dagger^{1}$, Franco MAGRI $\dagger^{2}$ and Marco PEDRONI $\dagger^{3}$ \\ $\dagger^{1}$ SISSA, Via Beirut 2/4, I-34014 Trieste, Italy \\ E-mail: falqui@sissa.it \\ $\dagger^{2}$ Dipartimento di Matematica e Applicazioni, Università di Milano-Bicocca \\ Via degli Arcimboldi 8, I-20126 Milano, Italy \\ E-mail:magri@vmimat.mat.unimi.it \\ $\dagger^{3}$ Dipartimento di Matematica, Università di Genova \\ Via Dodecaneso 35, I-16146 Genova, Italy \\ E-mail: pedroni@dima.unige.it
}

\begin{abstract}
We discuss the bihamiltonian geometry of the Toda lattice (periodic and open). Using some recent results on the separation of variables for bihamiltonian manifold, we show that these systems can be explicitly integrated via the classical Hamilton-Jacobi method in the so-called Darboux-Nijenhuis coordinates.
\end{abstract}

\section{Introduction}

In this paper we reexamine the classical $A_{n}$-Toda systems with the aim of showing that these lattices fall into a notable class of bihamiltonian integrable systems: those for which a distinguished set of coordinates (the so-called Darboux-Nijenhuis (DN) coordinates) allows the solution of the Hamilton-Jacobi equations associated with the Hamiltonian flows by means of an (additive) separation of variables (SoV) [16, 1]. In particular, we will show that such coordinates arise from the geometry of the Poisson pencil after a Hamiltonian reduction process on suitable symplectic leaves.

DN coordinates (see, e.g., [11]) can be naturally defined on a Poisson-Nijenhuis (PN) manifold [9], that is, on a $2 n$-dimensional manifold $\mathcal{M}$ endowed with a symplectic twoform $\omega$ and a $(1,1)$ torsion free tensor $N$ satisfying certain compatibility conditions. In [2] we present and discuss an intrinsic condition to characterize those Hamiltonian systems on $\mathcal{M}$ for which DN coordinates separate the corresponding Hamilton-Jacobi equations. Moreover, some of the connections between Hamiltonian hierarchies which satisfy a certain recursion property with respect to the tensor $N$, and Gel'fand-Zakharevich (GZ) systems [7] are investigated there. This paper is devoted to frame the Toda lattices into such a scheme.

The plan is as follows: in Section 2 we sketch the main points of the abovementioned SoV theory for bihamiltonian manifold, referring to [2] for complete proofs and a more detailed discussion. Section 3 contains a formulation of the Toda lattice within the GZ scheme, that is, taking as starting point its Poisson pencil and the problem of finding the 
Casimir functions. Section 4 concerns the application of the bihamiltonian SoV theory to this family of integrable systems. Finally, in Section 5 we treat the three-particle case to give a feeling of how the method works.

\section{Separation of variables on $\mathrm{PN}$ manifolds}

Let $(\mathcal{M}, \omega)$ be a $2 n$-dimensional symplectic manifold endowed with a Nijenhuis tensor field $N$ compatible with $\omega$ (in the sense of the theory of bihamiltonian manifolds). These manifolds are called Poisson-Nijenhuis manifolds [9]. Examples of such manifolds are provided by bihamiltonian manifolds endowed with a pair of Poisson bivectors $\left(P_{0}, P_{1}\right)$ one of which, say $P_{0}$, is invertible, In this case, $\omega=P_{0}^{-1}$ and $N=P_{1} P_{0}^{-1}$.

Definition 2.1. By Darboux-Nijenhuis coordinates on $\mathcal{M}$ we mean any system of local coordinates $\left(\lambda_{j}, \mu_{j}\right)_{j=1, \ldots, n}$ which enjoy the following two properties:

i) $\omega$ takes the canonical form

$$
\omega=\sum_{i=1}^{n} d \lambda_{i} \wedge d \mu_{i} ;
$$

ii) the adjoint Nijenhuis operator $N^{*}$ takes the diagonal form

$$
N^{*} d \lambda_{j}=\lambda_{j} d \lambda_{j}, \quad N^{*} d \mu_{j}=\lambda_{j} d \mu_{j} .
$$

It has been shown [11] that DN coordinates exist on any PN manifold, where $N$ has $n$ functionally independent eigenvalues. In this case the coordinates $\lambda_{j}$ can be computed algebraically as the roots of the minimal polynomial of $N$,

$$
C(\lambda)=\operatorname{Det}(N-\lambda \mathbf{1})^{\frac{1}{2}} .
$$

On the contrary, the complementary coordinates must be computed (in general) by a method involving quadratures.

In [2] we characterize a class of Hamiltonians on $\mathcal{M}$ whose associated Hamilton-Jacobi equations can be solved by separation of variables in DN coordinates. Let $\left(H_{1}, \ldots, H_{n}\right)$ be a set of functionally independent (Hamiltonian) functions that are in involution with respect to the canonical Poisson bracket defined by

$$
\{f, g\}=\omega\left(X_{f}, X_{g}\right) .
$$

We assume that the Lagrangian foliation defined by the functions $H_{i}$ is invariant with respect to $N$. This is tantamount to saying that, at a generic point $m \in \mathcal{M}$, the differentials $d H_{k}$ span an $n$-dimensional vector subspace of $T_{m}^{*} \mathcal{M}$ which is closed under the action of $N^{*}$. Hence there exists an $n \times n$ matrix $\mathrm{F}$, whose entries are functions on $\mathcal{M}$, such that

$$
N^{*} d H_{i}=\sum_{j=1}^{n} \mathrm{~F}_{i}^{j} d H_{j} .
$$

Definition 2.2. The Hamiltonians $\left(H_{1}, \ldots, H_{n}\right)$ are said to be Stäckel-separable (or separable for short) in the DN coordinates if there exists an $n \times n$ invertible matrix $\mathrm{T}$ and an $n$-component vector $V$ such that

$$
\mathrm{T} H=V,
$$


where $H=\left(H_{1}, \ldots, H_{n}\right)^{T}$, and the matrix $\mathrm{T}$ and the vector $V$ possess the Stäckel properties:

1. the entries of the $j^{\text {th }}$ row of $\mathrm{T}$ depend only on the conjugated coordinates $\left(\lambda_{j}, \mu_{j}\right)$.

2. the $j^{\text {th }}$ component of the vector $V$ depends only on $\left(\lambda_{j}, \mu_{j}\right)$ as well.

A remarkable "separability test" is given by the following

Theorem 2.3. The Hamiltonians $\left(H_{1}, \ldots, H_{n}\right)$ are separable if and only if the matrix $\mathrm{F}$ verifies the equation

$$
N^{*} d \mathrm{~F}=\mathrm{F} d \mathrm{~F} .
$$

Two remarks are in order to explain this theorem. First of all, equation (2.4) must be read as follows. In the left hand-side, $d \mathrm{~F}$ is the matrix whose entries are the differentials of the entries of $\mathrm{F}$, and $N^{*}$ acts separately on each entry. Secondly, one should notice that (2.4) is a coordinate free test of separability, that can be checked without computing the DN coordinates. Once the test is passed one can construct the Stäckel matrix T, still in general coordinates, by a simple algebraic procedure. One has to consider the eigenvectors of the matrix $\mathrm{F}$ and form with them a (suitably normalized) matrix $\mathrm{T}$ that diagonalizes $\mathrm{F}$ :

$$
\mathrm{F}=\mathrm{T}^{-1} \Lambda \mathrm{T}, \quad \Lambda=\operatorname{diag}\left(\lambda_{1}, \ldots, \lambda_{n}\right)
$$

By condition (2.4), this matrix is a Stäckel matrix; by condition (2.2), the vector $V=\mathrm{T} H$ verifies the Stäckel property. Then, once constructed the DN coordinates, the HamiltonJacobi equations associated with $\left(H_{1}, \ldots, H_{n}\right)$ can be easily solved by separation of variables. Notice that the DN coordinates separate at once the HJ equations associated with any of the Hamiltonians $H_{i}$.

To complete the construction of the DN coordinates, that is, to construct algebraically the coordinates $\mu_{j}$ conjugated to the eigenvalues $\lambda_{j}$ of $N$, the following procedure is often useful. We consider the Hamiltonian vector field $Y$ associated (by the symplectic form $\omega$ ) with the function $\frac{1}{2} \operatorname{Tr}(N)$, and the space of functions $F(x ; \lambda)$, depending smoothly on $x \in \mathcal{M}$ and holomorphically on the parameter $\lambda$. We denote with $F\left(x ; \lambda_{j}\right)$ the evaluation of $F(x ; \lambda)$ at $\lambda=\lambda_{j}$. If $N^{*} d F\left(x ; \lambda_{j}\right)=\lambda_{j} d F\left(x ; \lambda_{j}\right)$ for all $j=1, \ldots, n$, we say that $F(x ; \lambda)$ is an exact eigenvector of $N^{*}$.

Theorem 2.4. If $F(x ; \lambda)$ is an exact eigenvector of $N^{*}$, satisfying the "normalization property" $Y(F(x ; \lambda))=1$, then the evaluation of $F(x ; \lambda)$ at the points $\lambda=\lambda_{j}$, i.e.,

$$
\mu_{j}=F\left(x ; \lambda_{j}\right)
$$

provides a set of $n$ remaining $D N$ coordinates.

In the application of Section 4, we will use the property that if $F(x ; \lambda)$ is an exact eigenvector, then $Y(F(x ; \lambda))$ is an exact eigenvector as well. Since in the separable case a suitable combination of the Hamiltonians is exact, one can act with $Y$ on such a combination and generate a space of exact eigenvectors where the equation $Y(F(x ; \lambda))=1$ may be solved algebraically. 


\subsection{DN separable Hamiltonians from GZ systems}

Let $\mathcal{M}$ a $(2 n+k)$-dimensional manifold endowed with a pencil $P_{\lambda}=P_{1}-\lambda P_{0}$ of Poisson tensors. We suppose that it admits $k$ polynomial Casimir functions

$$
H^{(a)}=\sum_{j=0}^{n_{a}} H_{j}^{(a)} \lambda^{n_{a}-j}, \quad a=1, \ldots, k,
$$

with $n=n_{1}+\cdots+n_{k}$. If the functions $H_{j}^{(i)}$ are functionally independent, then $\mathcal{M}$ is called a complete GZ manifold, and the pencil $P_{\lambda}$ is said to be a pure Kronecker pencil of type $\left\{2 n_{1}+1, \ldots, 2 n_{k}+1\right\}$. Since the functions $H_{0}^{(a)}$ form a maximal set of independent Casimirs of $P_{0}$, the generic symplectic leaf $\mathcal{S}$ of $P_{0}$ is the $2 n$-dimensional submanifold given by $H_{0}^{(a)}=C_{a}$, for $a=1, \ldots, k$. The restrictions $\widehat{H}_{j_{a}}^{(a)}$ to $\mathcal{S}$, for $j_{a}=1, \ldots, n_{a}$, and $a=1, \ldots, k$, of the $n$ remaining Hamiltonians define a completely integrable system in the Liouville sense.

In order to solve by SoV this system, we suppose that there exist $k$ vector fields $Z_{a}$ (to be called transversal vector fields) spanning a $k$-dimensional integrable distribution $\mathcal{Z}$ and satisfying:

a) The normalized transversality condition: $\operatorname{Lie}_{Z_{a}}\left(H_{0}^{(b)}\right)=\delta_{a}^{b}$ for all $a, b=1, \ldots, k$;

b) The deformation condition for the Lie derivatives: $\operatorname{Lie}_{Z_{a}}\left(P_{\lambda}\right)=\sum_{b=1}^{k} Z_{b} \wedge Y_{a}^{b}$ for some vector fields $Y_{b}^{a}$;

c) The "flatness" condition: $\operatorname{Lie}_{Z_{a}}\left(\operatorname{Lie}_{Z_{b}}\left(H^{(c)}(\lambda)\right)\right)=0, \forall a, b, c$.

Conditions a) and b) imply that the distribution $\mathcal{Z}$ is transversal to the symplectic leaves of $P_{0}$, and that the functions vanishing along $\mathcal{Z}$ are a Poisson subalgebra with respect to the Poisson pencil $P_{\lambda}$. Then, as a consequence of the Marsden-Ratiu theorem [12], we have that:

Proposition 2.5. The Poisson pencil on $\mathcal{M}$ can be projected on the generic symplectic leaf $\mathcal{S}$ of $P_{0}$, so that $\mathcal{S}$ becomes a $P N$ manifold. The functions $\widehat{H}_{j_{a}}^{(a)}$, for $j_{a}=1, \ldots, n_{a}$, and $a=1, \ldots, k$, satisfy the condition (2.2), that is, there exists an $n \times n$ matrix $\mathrm{F}$ such that $N^{*} d \widehat{H}=\mathrm{F} \widehat{H}$, where $\widehat{H}$ is a column vector collecting the above functions.

Under the "flatness" condition c), one can show that equation (2.4) is satisfied, so that the reduced Hamiltonian system is separable in the DN coordinates. These coordinates may be computed from the geometry of $P_{\lambda}$, without actually performing the reduction process. In this case, in fact:

1. The minimal polynomial of the Nijenhuis tensor $N$ induced, according to the previous proposition, on the leaf $\mathcal{S}$ is the determinant of the matrix

$$
G(\lambda)=\left[\operatorname{Lie}_{Z_{a}}\left(H^{(b)}(\lambda)\right)\right]_{a, b=1, \ldots, k},
$$

that is, $\operatorname{det} G(\lambda)=0$ iff $\lambda=\lambda_{j}$;

2. The vector field $Y$ of Theorem 2.4 is given by $Y=\sum_{a=1}^{k} Y_{a}^{a}$; 
3. If $\left(1, \rho_{2}(\lambda), \ldots, \rho_{k}(\lambda)\right)$ satisfies

$$
\left(1, \rho_{2}(\lambda), \ldots, \rho_{k}(\lambda)\right) G(\lambda)=0 \quad \text { for } \lambda=\lambda_{j},
$$

then $H^{(1)}(\lambda)+\rho_{2}(\lambda) H^{(2)}(\lambda)+\cdots+\rho_{k}(\lambda) H^{(k)}(\lambda)$ is an exact eigenvector of $N^{*}$. Hence it can be used to find a normalized exact eigenvector, and therefore the $\mu_{j}$ coordinates.

Remark 2.6. The SoV theory for PN manifolds outlined above provides intrinsic and algorithmic recipes to check whether a given Liouville integrable system defined on a PN manifold can be separated in the DN coordinates. On the other hand, the conditions under which one obtains separable Hamiltonians from a GZ manifold are by no means algorithmic. In particular, the existence of the distribution $\mathcal{Z}$ (that is, of the vector fields $Z_{a}$ and $Y_{a}^{b}$ fulfilling the above three properties) must be checked (and guessed) case by case. In $[3,4]$ some GZ systems, obtained from stationary reductions of the Boussinesq and $\mathrm{KdV}$ hierarchies, are discussed along these lines. In the next sections we will apply the scheme herewith outlined to the Toda lattices.

\section{The Bihamiltonian approach to Toda lattices}

The phase space of the (complex, periodic) Toda lattice (see, e.g., [5]) with $n$ sites (particles) is the manifold $\mathcal{M}=\left(\mathbb{C}^{*}\right)^{n} \times \mathbb{C}^{n}$ parametrized by the Flaschka coordinates $\left\{a_{i}, b_{i}\right\}_{i=1, \ldots, n}$. We endow it with the Poisson pencil $P_{\lambda}$ defined as follows (see, e.g., [14] and references cited therein). It associates with the one-form $\sum_{k}\left(\alpha_{k} d a_{k}+\beta_{k} d b_{k}\right)$ the vector field $\sum_{k}\left(\dot{a}_{k} \partial_{a_{k}}+\dot{b}_{k} \partial_{b_{k}}\right)$ according to the rule

$$
\begin{aligned}
\dot{a_{k}} & =a_{k}\left(\left(b_{k}-\lambda\right) \beta_{k}-\left(b_{k+1}-\lambda\right) \beta_{k+1}+a_{k-1} \alpha_{k-1}-\alpha_{k+1} a_{k+1}\right), \\
\dot{b_{k}} & =\left(b_{k}-\lambda\right)\left(a_{k-1} \alpha_{k-1}-a_{k} \alpha_{k}\right)+a_{k} \beta_{k+1}-a_{k-1} \beta_{k-1},
\end{aligned}
$$

where the cyclicity condition $(\cdot)_{k+n}=(\cdot)_{k}$ is implicitly assumed. We write the matrix expression of $P_{\lambda}=P_{1}-\lambda P_{0}$ in the 3 -particle case, the $n$-particle case being easily generalized from this example:

$$
P_{\lambda}=\left[\begin{array}{cccccc}
0 & -a_{1} a_{2} & a_{1} a_{3} & a_{1}\left(b_{1}-\lambda\right) & -a_{1}\left(b_{2}-\lambda\right) & 0 \\
& 0 & -a_{2} a_{3} & 0 & a_{2}\left(b_{2}-\lambda\right) & -a_{2}\left(b_{3}-\lambda\right) \\
& & 0 & -a_{3}\left(b_{1}-\lambda\right) & 0 & a_{3}\left(b_{3}-\lambda\right) \\
& & 0 & a_{1} & -a_{3} \\
& * & & 0 & a_{2} \\
& & & & & 0
\end{array}\right] .
$$

According to the GZ scheme, we study the kernel of $P_{\lambda}$. We have to solve the equations

$$
\begin{aligned}
& \left(b_{k}-\lambda\right) \beta_{k}-\left(b_{k+1}-\lambda\right) \beta_{k+1}+a_{k-1} \alpha_{k-1}-a_{k+1} \alpha_{k+1}=0, \\
& \left(b_{k}-\lambda\right)\left(a_{k-1} \alpha_{k-1}-a_{k} \alpha_{k}\right)+a_{k} \beta_{k+1}-a_{k-1} \beta_{k-1}=0 .
\end{aligned}
$$

With algebraic manipulations (see [13]), it can be traded for the system of equations

$$
\begin{aligned}
& \left(b_{k}-\lambda\right) \beta_{k}+a_{k-1} \alpha_{k-1}+a_{k} \alpha_{k}=L_{1}, \\
& \left(a_{k} \alpha_{k}\right)^{2}+a_{k} \beta_{k} \beta_{k+1}-L_{1} \alpha_{k}=L_{2},
\end{aligned}
$$


where $L_{i}$ are $\mathbb{Z}_{n}$-invariant functions. Setting $L_{1}=1, L_{2}=0$, and introducing the variables

$$
h_{k}=\frac{\beta_{k+1}}{\alpha_{k}},
$$

we obtain the following Riccati type equation:

$$
h_{k} h_{k+1}=\left(b_{k+1}-\lambda\right) h_{k}+a_{k} .
$$

Proposition 3.1. The characteristic equation (3.4) admits a solution $h_{k}$ which is a Laurent series in the parameter $\lambda$ of the form $h_{k}=\lambda+\sum_{j=1}^{\infty} h_{k, j} \lambda^{-j}$. The Laurent coefficients $h_{k, j}$ can be computed by recurrence as functions of the variables $\left\{a_{i}, b_{i}\right\}$. The product $C=h_{1} \cdots h_{n}$ of the components of any solution of (3.4) is a Casimir function of the Poisson pencil $P_{\lambda}$.

Notice that, once the characteristic equation is solved, the one-forms in the kernel of $P_{\lambda}$ can be easily computed (by recurrence) solving the system

$$
\left\{\begin{array}{l}
h_{k} \alpha_{k}+a_{k} \beta_{k}=1, \\
\alpha_{k} h_{k}=\beta_{k+1},
\end{array} \quad k=1, \ldots, n,\right.
$$

which is equivalent to the system (3.3) with $L_{1}=1, L_{2}=0$.

This method allows us to find Casimirs of $P_{\lambda}$ that are Laurent series in $\lambda$. According to the GZ scheme [7], however, we should better look for polynomial Casimirs of $P_{\lambda}$. They can be found linearizing the Riccati equation (3.4) as follows.

Setting $h_{k}=\mu \psi_{k} / \psi_{k-1}$, we transform equation (3.4) into the linear system

$$
\mu^{2} \psi_{k+1}-\mu\left(b_{k}-\lambda\right) \psi_{k}-a_{k} \psi_{k-1}=0,
$$

where $\mu$ is related to the Casimir $C$ via $C=\mu^{n}$. In matrix form we have $\mathcal{L} \psi=0$, where

$$
\mathcal{L}=\left[\begin{array}{ccccc}
\mu\left(b_{1}-\lambda\right) & -\mu^{2} & 0 & & a_{n} \\
a_{1} & \mu\left(b_{2}-\lambda\right) & -\mu^{2} & \ddots & \\
0 & a_{2} & \ddots & \ddots & 0 \\
& \ddots & \ddots & \mu\left(b_{n-1}-\lambda\right) & -\mu^{2} \\
-\mu^{2} & 0 & & a_{n-1} & \mu\left(b_{n}-\lambda\right)
\end{array}\right]
$$

This is how the classical Lax matrix of the Toda lattice can be introduced into the game in the GZ bihamiltonian point of view. We remark that, since the Riccati equation (3.4) admits solutions, so does the linear system $\mathcal{L} \psi=0$. So, taking into account the cyclicity of $\mathcal{L}$, we arrive at

Proposition 3.2. The spectral curve of the problem, $\operatorname{det}(\mathcal{L})=0$, is a quadratic polynomial in the Casimir $C$,

$$
\operatorname{det}(\mathcal{L})=-C^{2}+H^{(1)}(\lambda) C+H^{(2)} .
$$

Thus, both $H^{(1)}(\lambda)$ and $H^{(2)}$ are polynomial Casimirs of $P_{\lambda}$. In particular,

$$
H^{(2)}=(-1)^{n+1} a_{1} \cdots a_{n}
$$


is a common Casimir of $P_{1}$ and $P_{0}$, and $H^{(1)}(\lambda)$ has the form

$$
H^{(1)}(\lambda)=(-1)^{n} \lambda^{n}+\sum_{j=1}^{n} H_{j}^{(1)} \lambda^{n-j} .
$$

It can be easily realized that $H_{j}^{(1)}=(-1)^{j} \sigma_{j}^{n}\left(b_{1}, \ldots, b_{n}\right)$ +lower order terms in the $b_{j}$, where $\sigma_{j}^{n}$ is the $j$-th elementary symmetric polynomial in $n$ letters. So, the Hamiltonian

functions $\left(H^{(2)},\left\{H_{j}^{(1)}\right\}_{j=1, \ldots, n}\right)$ are functionally independent and the previous proposition provides another proof of the fact [8] that the periodic Toda lattice with $n$ particles is a complete GZ manifold of type $\{1,2 n-1\}$.

We end this section with a remark which frames the open Toda lattice within this scheme. It is well known that the open Toda lattice can be obtained form the periodic one by pulling one particle to infinity, that is, in the Flaschka coordinates, by letting one of the $a$ coordinates, say $a_{n}$, attain the 0 value. The phase space of the (complex) open Toda lattice with $n$ particles is thus the manifold $\widehat{\mathcal{M}}=\left(\mathbb{C}^{*}\right)^{n-1} \times \mathbb{C}^{n}$ parametrized by reduced Flaschka coordinates $\left\{a_{1} \ldots, a_{n-1}, b_{1}, \ldots, b_{n}\right\}$. The Poisson pencil $\widehat{P_{\lambda}}$ of the open case can be obtained from the periodic one by means of the following trick. Let $\widetilde{\mathcal{M}} \supset \mathcal{M}$ be the manifold obtained from the phase space of the periodic lattice adjoining the "boundary component" defined by $a_{n}=0$. The Poisson pencil defined by (3.1), being polynomial in the Flaschka coordinates, extends naturally to a Poisson pencil $\widetilde{P}_{\lambda}$ on the extended manifold $\widetilde{\mathcal{M}}$. The phase space $\widehat{\mathcal{M}}$ of the open case can be identified with the zero set of the common Casimir $H^{(2)}$ of $\widetilde{P}_{1}$ and $\widetilde{P}_{0}$, which, obviously enough, is still given by $H^{(2)}=(-1)^{n+1} a_{1} \cdots a_{n}$. Then $\widetilde{P}_{\lambda}$ can be restricted to $\widehat{\mathcal{M}}$, and its restriction is the Poisson pencil of the open Toda lattice. In practice, its matrix representation in the reduced coordinates is obtained by the matrix representation of the periodic Poisson pencil (3.2) deleting the $n$-th row and column, and setting $a_{n}=0$ in the resulting matrix. The Lax matrix of the open Toda Lattice (as well as the Hamiltonian functions) is obtained simply by setting $a_{n}=0$ in the Lax matrix (3.6) of the periodic problem. In particular, the single polynomial Casimir of the Poisson pencil of the open lattice is obtained as $\widehat{H}^{(1)}=H^{(1)}{ }_{a_{n}=0}$. The open Toda lattice is thus a complete GZ manifold of type $2 n-1$.

\section{Separation of variables}

In this Section we will show that the Toda lattice fits the scheme described in Subsection 2.1. We will follow the path of Section 3, considering at first the periodic lattice, and then stating the suitable changes to be done in the open case.

The periodic Toda lattice is a GZ manifold of dimension $2 n$ and type $\{1,2 n-1\}$. Thus the rank of the transversal distribution $\mathcal{Z}$ must be 2 , and the dimension of the reduced PN manifold $2 n-2$. We divide the procedure outlined in Subsection 2.1 in three steps.

Step 1. The transversal vector fields $Z_{1}$ and $Z_{2}$.

Proposition 4.1. The vector fields $Z_{1}=\partial_{b_{n}}$ and $Z_{2}=\partial_{a_{n}} /\left(a_{1} \cdots a_{n-1}\right)$ satisfy

$$
\operatorname{Lie}_{Z_{1}} P_{\lambda}=Z_{1} \wedge Y_{1,1}, \quad \operatorname{Lie}_{Z_{2}} P_{\lambda}=Z_{1} \wedge Y_{2,1},
$$

with $Y_{1,1}=a_{n-1} \partial_{a_{n-1}}-a_{n} \partial_{a_{n}}$ and $Y_{2,1}=-\partial_{b_{1}} /\left(a_{1} \cdots a_{n-1}\right)$. 
This property is proven making use of the standard formulas for the Lie derivative of a bivector.

Step 2. The action of $Z_{i}$ on the Casimirs and the $\lambda_{j}$ coordinates.

To discuss this issue it is useful to recall the expression of the second Casimir $H^{(2)}=$ $(-1)^{n+1} a_{1} \cdots a_{n}$ and to expand $\operatorname{det}(\mathcal{L})$ with respect to the last column to get:

$$
\operatorname{det}(\mathcal{L})=\mu\left(b_{n}-\lambda\right) \widehat{L}_{n, n}+\mu^{2} \widehat{L}_{n, n-1}+(-1)^{n+1} a_{n} \widehat{L}_{n, 1},
$$

where $\widehat{L}_{i, j}$ are the determinants of the suitable minors of $\mathcal{L}$. Taking into account the specific form of these minors one can easily see that it holds

Proposition 4.2. The second Lie derivatives of $H^{(1)}$ and $H^{(2)}$ with respect to $Z_{i}$ vanish. Furthermore, $\operatorname{Lie}_{Z_{1}}\left(H^{(2)}\right)=0$ and $\operatorname{Lie}_{Z_{2}}\left(H^{(2)}\right)=1$, so that the matrix $G_{a}^{b}=$ $\operatorname{Lie}_{Z_{a}}\left(H^{(b)}(\lambda)\right)$ introduced in Subsection 2.1 has the form

$$
G(\lambda)=\left(\begin{array}{ll}
\operatorname{Lie}_{Z_{1}}\left(H^{(1)}\right) & 0 \\
\operatorname{Lie}_{Z_{2}}\left(H^{(1)}\right) & 1
\end{array}\right)
$$

Thus the $\lambda_{j}$ coordinates are the roots of the monic degree $(n-1)$ polynomial $\operatorname{Lie}_{Z_{1}}\left(H^{(1)}\right)=$ $\partial_{b_{n}} \operatorname{det}(\mathcal{L})=\widehat{L}_{n, n}$.

Step 3. The action of the vector field $Y$ and the $\mu_{j}$ coordinates.

We have to consider the vector field $Y=Y_{1,1}=a_{n-1} \partial_{a_{n-1}}-a_{n} \partial_{a_{n}}$, and to discuss its action on the exact eigenvectors of $N^{*}$. According to the discussion following Proposition 2.5, to construct such an eigenvector we must find a vector $(1, \rho(\lambda))$ such that $(1, \rho(\lambda)) G(\lambda)=0$ for $\lambda=\lambda_{j}$. Since this vector is simply given by $(1,0)$, we have that $H^{(1)}$ is an exact eigenvector of $N^{*}$, and this is true, for all $r$, for $Y^{r}\left(H^{(1)}\right)$ as well. In order to build a normalized exact eigenvector, we have to analyze a bit further the terms in the expansion (4.2) of the determinant of $\mathcal{L}$. Actually, one has that:

1. $\widehat{L}_{n, n}$ is independent of $a_{n}$ and $a_{n-1}$;

2. $a_{n} \widehat{L}_{n, 1}=H^{(2)}+C K_{1}$, where $K_{1}$ is linear in $a_{n}$ and does not depend on $a_{n-1}$ and $\mu$;

3. $\mu^{2} \widehat{L}_{n, n-1}=C^{2}+C K_{2}$, where $K_{2}$ is linear in $a_{n-1}$ and does not depend on $a_{n}$ and $\mu$.

Thanks to the linearity properties of $K_{j}$, we have that $Y\left(H^{(1)}\right)=Y\left(K_{1}+K_{2}\right)$ satisfies the recursion property $Y^{3}\left(H^{(1)}\right)=Y\left(H^{(1)}\right)$. This ensures that the function

$$
F=\log \left(Y\left(H^{(1)}\right)+Y^{2}\left(H^{(1)}\right)\right)
$$

satisfies $Y(F)=1$, and, according to Theorem 2.4, is the desired generator of the $\mu_{j}$ coordinates. We notice that, due to the cyclic nature of the periodic Toda system, the pair $Z_{1}, Z_{2}$ of deformation vector fields is by no means unique; other admissible pairs can be obtained via a cyclic permutation of the indices (with a corresponding change in the vector field $Y$ ). It would be interesting to compare the DN coordinates considered here with the separation variables for Toda systems used in, e.g., [6, 10, 15].

Finally, we state the corresponding results for the open Toda lattice. We have to look for a single deformation vector field, say $Z$; it is still given by $\partial_{b_{n}}$; the vector field $Y$ now is given by $a_{n-1} \partial_{a_{n-1}}$; the recursion relation on $Y\left(\widehat{H}^{(1)}\right)$ closes at the first step, $Y^{2}\left(\widehat{H}^{(1)}\right)=Y\left(\widehat{H}^{(1)}\right)$, and the generating function can be taken as $\widehat{F}=\log \left(Y\left(\widehat{H}^{(1)}\right)\right)$. 


\section{Example: the three-particle case}

We close the paper with some explicit expressions for the three-particle case. The Poisson pencil is written in equation (3.2). The Lax matrix is given by

$$
\mathcal{L}=\left(\begin{array}{ccc}
\mu\left(b_{1}-\lambda\right) & -\mu^{2} & a_{3} \\
a_{1} & \mu\left(b_{2}-\lambda\right) & -\mu^{2} \\
-\mu^{2} & a_{2} & \mu\left(b_{3}-\lambda\right)
\end{array}\right) .
$$

The spectral curve is

$$
-C^{2}-\left(\lambda^{3}+H_{0} \lambda^{2}+H_{1} \lambda+H_{2}\right) C+K=0,
$$

with

$$
\begin{aligned}
& H_{0}=-\left(b_{1}+b_{3}+b_{2}\right), \quad H_{1}=a_{2}+b_{1} b_{3}+a_{1}+b_{2} b_{3}+a_{3}+b_{1} b_{2}, \\
& H_{2}=-\left(b_{1} b_{2} b_{3}+a_{1} b_{3}+b_{1} a_{2}+a_{3} b_{2}\right), \quad K=a_{1} a_{2} a_{3}, \quad C=\mu^{3} .
\end{aligned}
$$

There are two nontrivial flows, given by:

$$
\begin{aligned}
& X_{1}=\left\{\begin{array}{l}
\dot{a}_{1}=a_{1} b_{2}-a_{1} b_{1}, \\
\dot{b}_{1}=a_{3}-a_{1}, \\
\text { and cyclic permutations; }
\end{array}\right. \\
& X_{2}=\left\{\begin{array}{l}
\dot{a}_{1}=a_{1} b_{1} b_{3}+a_{1} a_{3}-a_{1} b_{2} b_{3}-a_{1} a_{2}, \\
\dot{b}_{1}=a_{1} b_{3}-a_{3} b_{2}, \\
\text { and cyclic permutations. }
\end{array}\right.
\end{aligned}
$$

The transversal vector fields are $Z_{1}=\partial_{b_{3}}$ and $Z_{2}=\partial_{a_{3}} / a_{1} a_{2}$, and we have $Y=a_{2} \partial_{a_{2}}-$ $a_{3} \partial_{a_{3}}$. The DN coordinates can be found as follows. The roots of the polynomial

$$
\operatorname{Lie}_{Z_{1}}\left(H^{(1)}(\lambda)\right)=\partial_{b_{3}}\left(H_{0} \lambda^{2}+H_{1} \lambda+H_{2}\right)=-\lambda^{2}+\left(b_{1}+b_{2}\right) \lambda-\left(b_{1} b_{2}+a_{1}\right)
$$

are $\lambda_{1}$ and $\lambda_{2}$. Then $\mu_{1}$ and $\mu_{2}$ are given by the function

$$
F=\log \left(Y\left(H^{(1)}\right)+Y^{2}\left(H^{(1)}\right)\right)=\log \left(2 a_{2} \lambda-2 a_{2} b_{1}\right)
$$

evaluated at $\lambda=\lambda_{1}, \lambda_{2}$.

For the open case, the Poisson pencil can be computed from (3.2), according to the procedure outlined at the end of Section 3:

$$
P_{\lambda}^{\text {open }}=\left[\begin{array}{ccccc}
0 & -a_{1} a_{2} & \left(b_{1}-\lambda\right) a_{1} & \left(\lambda-b_{2}\right) a_{1} & 0 \\
a_{1} a_{2} & 0 & 0 & \left(b_{2}-\lambda\right) a_{2} & \left(\lambda-b_{3}\right) a_{2} \\
\left(\lambda-b_{1}\right) a_{1} & 0 & 0 & a_{1} & 0 \\
\left(b_{2}-\lambda\right) a_{1} & \left(\lambda-b_{2}\right) a_{2} & -a_{1} & 0 & a_{2} \\
0 & \left(b_{3}-\lambda\right) a_{2} & 0 & -a_{2} & 0
\end{array}\right],
$$

and the spectral curve is the rational curve

$$
\begin{aligned}
\mu^{3}=C= & -\lambda^{3}+\left(b_{2}+b_{1}+b_{3}\right) \lambda^{2}-\left(b_{1} b_{2}+a_{1}+b_{2} b_{3}+b_{1} b_{3}+a_{2}\right) \lambda \\
& +b_{1} b_{2} b_{3}+a_{1} b_{3}+b_{1} a_{2} .
\end{aligned}
$$

The separation variables can be constructed, mutatis mutandis, as in the periodic case. 


\section{Acknowledgements}

G.F. and M.P. would like to thank the organizers of NEEDS99 for the opportunity to present these results there, and for financial support to their participation. This work was partially supported by the M.U.R.S.T. and the G.N.F.M. of the Italian C.N.R.

\section{References}

[1] Adams M R, Harnad J and Hurtubise J, Darboux Coordinates and Liouville-Arnold Integration in Loop Algebras, Comm. Math. Phys., 1993, V.155, 385-413.

[2] Falqui G, Magri F and Pedroni M (in preparation).

[3] Falqui G, Magri F, Pedroni M and Zubelli J P, A Bi-Hamiltonian Theory for Stationary KdV Flows and Their Separability, Regular and Chaotic Dynamics, 2000, V.5, 33-51.

[4] Falqui G, Magri F and Tondo G, Reduction of Bihamiltonian Systems and Separation of Variables: an Example from the Boussinesq Hierarchy, Theor. Math. Phys., 2000, V.122, $212-230$.

[5] Flaschka H, Integrable Systems and Torus Actions, in Lectures on Integrable Systems, Editors O Babelon et al., World Scientific, 1994, 43-101.

[6] Flaschka H and McLaughlin D W, Canonically Conjugate Variables for the Korteweg-de Vries Equation and the Toda Lattice with Periodic Boundary Conditions, Progress Theor. Phys., 1976, V.55, 438-456.

[7] Gel'fand I M and Zakharevich I, On the Local Geometry of a Bi-Hamiltonian Structure, in The Gel'fand Mathematical Seminars 1990-1992, Editors L Corwin et al., Birkhäuser, Boston, 1993, 51-112.

[8] Gel'fand I M and Zakharevich I, Webs, Lenard Schemes, and the Local Geometry of Bihamiltonian Toda and Lax Structures, math-ag/9903080, Selecta Mathematica (to appear).

[9] Kosmann-Schwarzbach Y and Magri F, Poisson-Nijenhuis Structures, Ann. Inst. Poincaré (Phys. Theor.), 1990, V.53, 35-81.

[10] Kuznetsov V B, Separation of Variables for the $D_{n}$-Type Periodic Toda Lattice, J. Phys. A, 1997, V.30, 2127-2138.

[11] Magri F, Geometry and Soliton Equations, in La Mécanique Analytique de Lagrange et son héritage, Atti Acc. Sci. Torino Suppl., 1990, V.124, 181-209.

[12] Marsden J E and Ratiu T, Reduction of Poisson Manifolds, Lett. Math. Phys., 1986, V.11, $161-169$.

[13] Meucci A, The Bihamiltonian Route to the Discrete Sato Grassmannian, Ph. D. Thesis, Dipartimento di Matematica dell'Università di Milano, 1998.

[14] Morosi C and Pizzocchero L, R-Matrix Theory, Formal Casimirs and the Periodic Toda Lattice, J. Math. Phys., 1996, V.37, 4484-4513.

[15] Sklyanin E K, The Quantum Toda Chain, in Nonlinear Equations in Classical and Quantum Field Theory, Meudon/Paris, 1983/1984, Lecture Notes in Phys. 226, Springer, Berlin, 1985, $196-233$.

[16] Sklyanin E K, Separations of Variables: New Trends, Progr. Theor. Phys. Suppl., 1995, V.118, $35-60$. 